\title{
1+ / N+ method: numerical simulation studies and experimental measurements on the SPIRAL1 ECR Charge Breeder
}

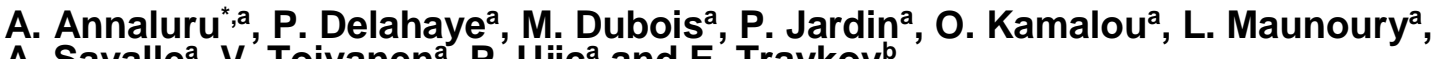 \\ A. Savalle ${ }^{\mathrm{a}}$, V. Toivanen ${ }^{\mathrm{a}}, \mathrm{P}$. Ujic ${ }^{\mathrm{a}}$ and E. Traykov \\ ${ }^{a}$ Grand Accélérateur National d'Ions Lourds (GANIL), \\ Boulevard Henri Becquerel, 14000 Caen, France \\ ${ }^{b}$ Institut pluridisciplinaire Hubert Curien (IPHC), \\ 23 Rue du Loess, 67200 Strasbourg, France \\ E-mail: arun.annaluru@ganil.fr
}

ABSTRACT: In the framework of the SPIRAL1 facility, the R \& D of charge breeding technique is of primary interest for optimizing the yields of radioactive ion beams (RIBs). This technique involves the transformation of mono-charged ion beams into multi-charged ion beams by operating an Electron Cyclotron Resonance (ECR) charge breeder (CB). During the SPIRAL1 commissioning, experimental studies have been performed in order to understand the transport of the beam through the CB with and without ECR plasma. Numerical simulations including ion optics and some ECR plasma features have been developed to evaluate ion losses during the ion transport through the $\mathrm{CB}$ with and without a simplified model of the ECR plasma.

KEYWORDS: Beam Optics; ECR Ion Sources; Accelerator modeling and simulations.

\footnotetext{
* Corresponding author.
} 


\section{Contents}

1. Introduction

2. Experimental activities with SPIRAL1 ECR Charge Breeder 2

2.1 Modification of axial magnetic field 2

2.2 Transmission efficiencies of ${ }^{39} \mathrm{~K}^{+}$and ${ }^{23} \mathrm{Na}^{+} \quad 3$

2.3 Charge breeding of ${ }^{23} \mathrm{Na} \quad 4$

2.4 Effect of deceleration tube position on charge breeding efficiency 4

2.5 Effect of injected beam emittance on charge breeding efficiency 5

3. Numerical simulation studies on ECR charge breeder 6

$\begin{array}{ll}3.1 \text { Simulation description } & 6\end{array}$

3.2 Ion transport through the charge breeder without plasma $\quad 6$

3.3 Ion transport through the Charge Breeder plasma $\quad 7$

4. Simulation Results and discussion $\quad 8$

$\begin{array}{lr}\text { Summary and perspectives } & 9\end{array}$

References $\quad 10$

\section{Introduction}

The SPIRAL1 facility at GANIL is dedicated to produce and accelerate the stable and radioactive ion beams which are delivered to physicists for nuclear experimental studies. Extending their research on exotic nuclei properties far from stability, an upgrade has been undertaken at SPIRAL1 to extend the range of post-accelerated exotic beams as well as to provide low energy $(\mathrm{keV})$ radioactive beams to the future DESIR (Désintégration, Excitation et Stockage d'Ions Radioactifs) experimental area. Different Target Ion Source System (TISS) based on a forced electron beam induced arc discharge (FEBIAD) type mono-charged ion source [1] has been chosen to provide $1+$ beams of condensable elements with high efficiency. The 1+ beams from TISS are mass analysed and transported to the $14.5 \mathrm{GHz}$ SPIRAL1 ECR charge breeder (CB), which increases the charge state charge of the radioactive ions from $1+$ to $\mathrm{N}+$. The extracted high charge state ions are mass analysed and post accelerated to CIME cyclotron (Cyclotron d'Ions de Moyenne Energie) [2].

Figure 1 shows the 3D cross-section of the charge breeder placed in the Low Energy Beam Transport (LEBT) between the TISS and the CIME. In the injection side of the CB the beam is focused with an electrostatic quadrupole triplet and the extracted beam is focused with an einzel lens. The charge breeder has gone through several modifications which are reported in [3], and experimental charge breeding results are presented in [4]. During its operation, a few technical changes have been done in the configuration of the charge breeder: the position of two soft iron rings has been modified to optimize the axial magnetic field and the optimization of the deceleration tube position to study its effect on beam stability and charge breeding efficiency. 


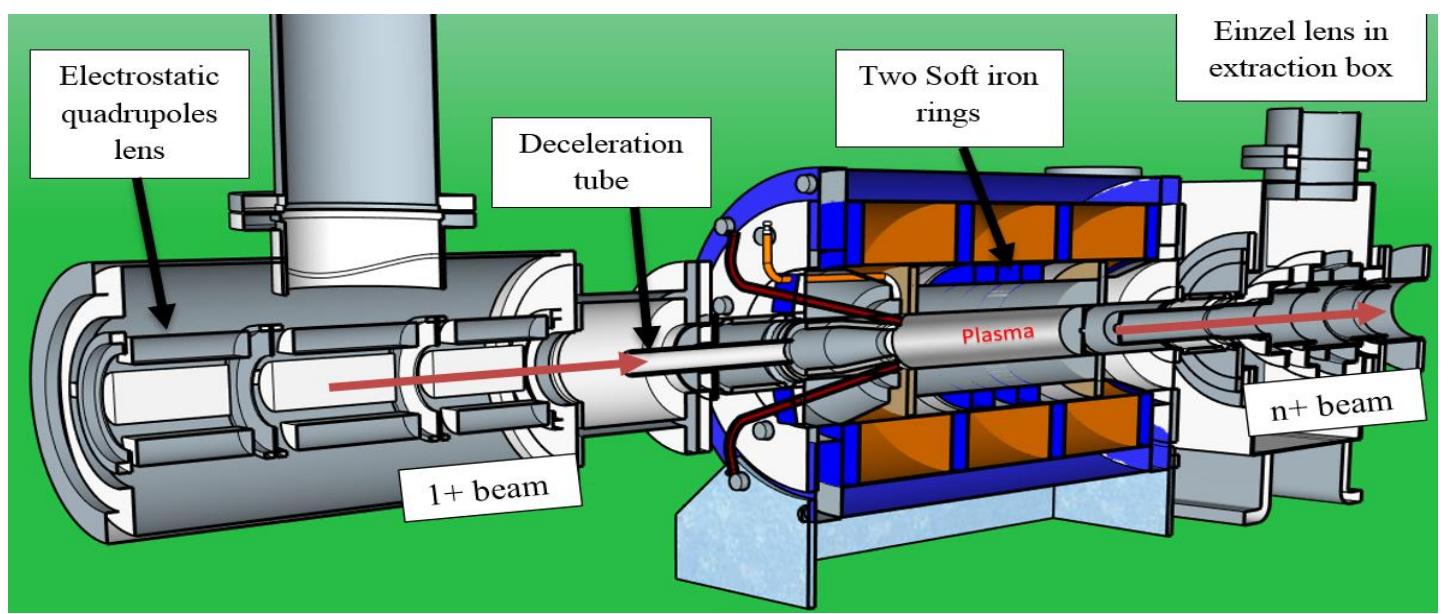

Figure 1. 3D view of ECR charge breeder installed between injection and extraction systems in LEBT.

Experimental studies on LEBT transport and charge breeding efficiencies for ${ }^{23} \mathrm{Na}^{+}$and ${ }^{23} \mathrm{Na}^{2+}$ have been carried out to understand the ion losses and 1+ capture processes by ECR plasma. A rough analysis on the experimental studies is described in the following sections by introducing a simplified ECR plasma model utilizing SIMION 3D. The results obtained from the simulations are presented and compared with the experimental ones.

\section{Experimental activities with SPIRAL1 ECR Charge Breeder}

\subsection{Modification of axial magnetic field}

In ECR ion source based charge breeders, the "Minimum-B" magnetic field structure is created by 2 or 3 independent solenoids (axial field) and hexapole (radial field) magnets enclosed in an iron yoke. In this configuration, the magnetic field is minimum at the centre of plasma chamber and increases in all directions. The Minimum-B configuration is essential to form a closed resonance surface in the plasma chamber where ECR condition is fulfilled.

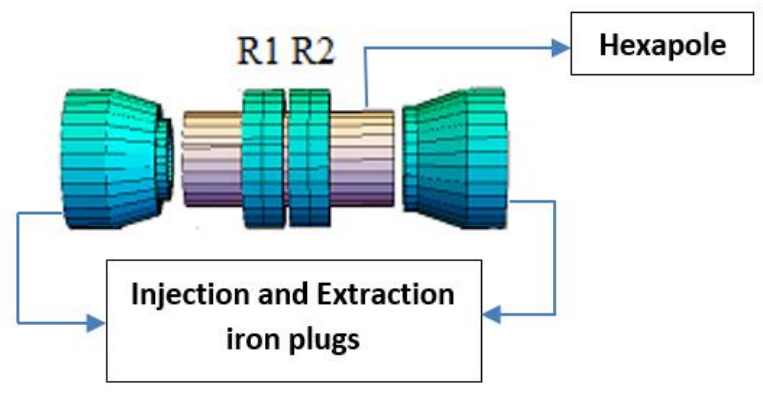

Figure 2. Position of two soft iron rings (R1 and R2) around the hexapole.

In SPIRAL1 charge breeder, the axial magnetic field $(\mathbf{B})$ can be adjusted with the center coil and two movable soft iron rings (R1 and R2) placed around the hexapole as shown in Figure 2. Magnetic field calculations were performed with RADIA [5] to study the axial magnetic field profile by adjusting the rings to different positions and significant variations were observed in the size of ECR zone in axial direction. Axial magnetic field profiles for different rings position are shown in Figure 3. 


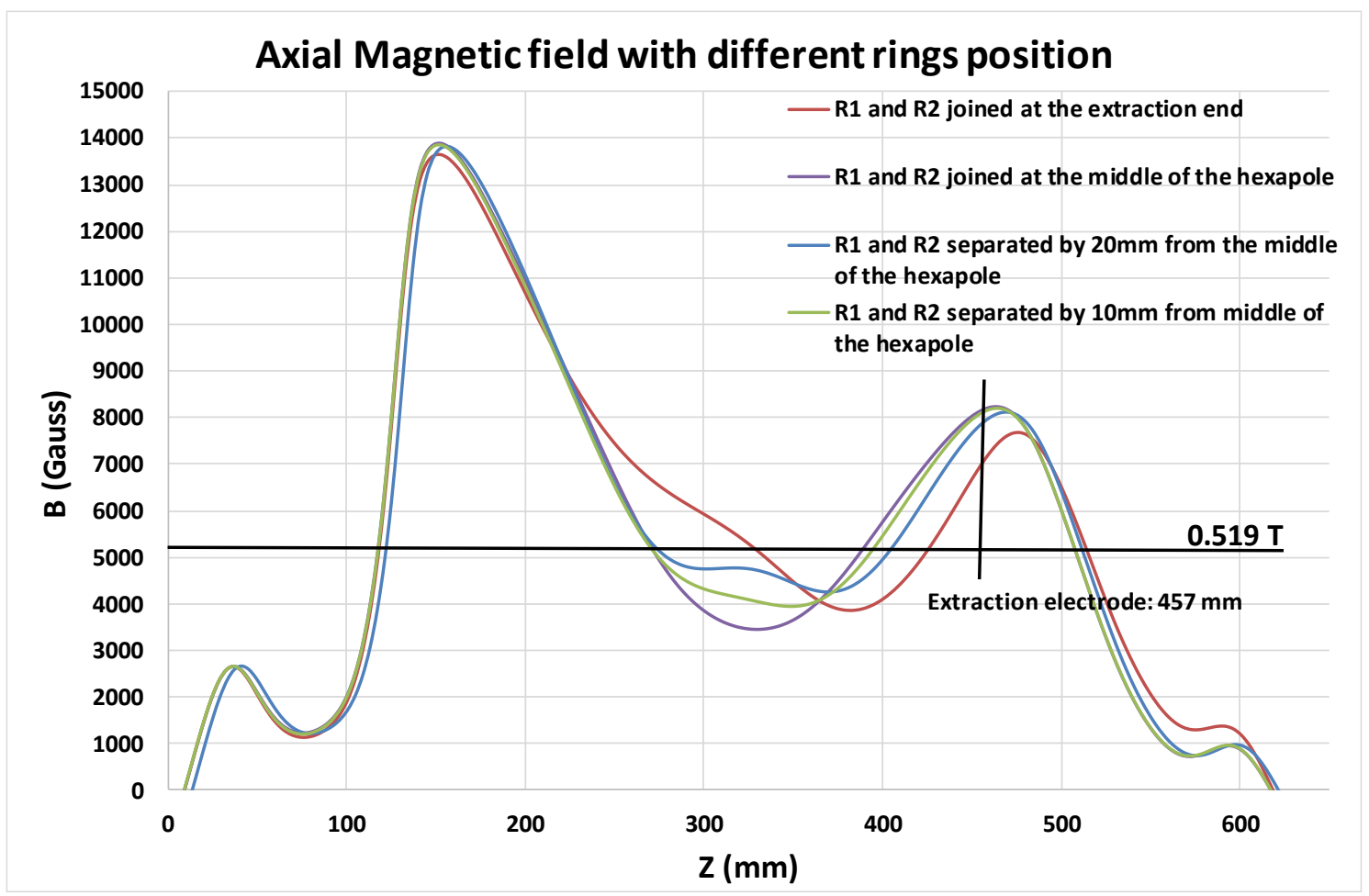

Figure 3. Axial $B$ fields with different positions of the two soft iron rings. a) Two rings joined at the extraction side of CB (Red) b) Two rings joined at the middle of the hexapole (purple) c) rings separated by $10 \mathrm{~mm}$ from the middle of the hexapole (Blue) d) rings separated by $20 \mathrm{~mm}$ from the middle of the hexapole (Red). Center of the hexapole refers to the center of plasma chamber $(320 \mathrm{~mm})$.

Charge breeding efficiency measurements were performed to study $1+/ \mathrm{N}+$ with potassium beam. Helium was used as buffer gas in CB. During these measurements, the soft iron rings (R1 and R2) were initially pushed towards the extraction end of $\mathrm{CB}$ and the charge breeding efficiency of $7.44 \%$ has been recorded for ${ }^{39} \mathrm{~K}^{9+}$. Later, the rings were adjusted to different positions around the hexapole. Best CB efficiency has been found when the rings R1 and R2 were separated by $10 \mathrm{~mm}$ from the middle of the hexapole. Table 1 shows the charge breeding efficiency of ${ }^{39} \mathrm{~K}^{9+}$ with different positions of the two iron rings. As a result, with this configuration charge breeding efficiency of ${ }^{39} \mathrm{~K}^{9+}$ has been increased by a factor of 1.5 when compared with initial position of the rings.

Table 1. ${ }^{39} \mathrm{~K}^{9+}$ Efficiency measured at different rings positions. Middle of the hexapole is referred as centre of plasma chamber $(320 \mathrm{~mm})$.

\begin{tabular}{|l|c|}
\hline \multicolumn{1}{|c|}{ Rings Position } & ${ }^{39} \mathbf{K}^{\mathbf{9}}$ efficiency \\
\hline R1 and R2 joined at extraction end & $7.44 \%$ \\
\hline R1 and R2 joined at the middle of hexapole & $10.08 \%$ \\
\hline R1 and R2 separated by 20 mm from middle of the hexapole & $10.88 \%$ \\
\hline R1 and R2 separated by 10 mm from middle of the hexapole & $11.8 \%$ \\
\hline
\end{tabular}

\subsection{Transmission efficiencies of ${ }^{39} \mathrm{~K}^{+}$and ${ }^{23} \mathrm{Na}^{+}$}

The 1+ and $n+$ beam lines have been tuned to achieve maximum ion beam current, up to the Faraday cup $\left(\mathrm{FC}_{1+}\right)$ and likewise to the Faraday cup $\left(\mathrm{FC}_{\mathrm{n}+}\right)$, which is placed after the charge 
breeder. Using TraceWin code [6], the beam transport is simulated with potassium and sodium beams in order to achieve high transmission efficiencies in LEBT. $\mathrm{Na}^{+}$and $\mathrm{K}^{+}$beams were produced and injected into the charge breeder with the typical intensities of 300 to $400 \mathrm{nA}$. By turning OFF the charge breeder (without plasma and high voltage), the transmission efficiency of $80 \%$ and $78 \%$ on ${ }^{39} \mathrm{~K}^{+}$and ${ }^{23} \mathrm{Na}^{+}$were recorded at faraday cup $\left(\mathrm{FC}_{\mathrm{n}+}\right)$. The axial $\mathrm{B}$ field configuration was: at injection $\mathbf{B}_{\text {inj }}=1.41 \mathrm{~T}$, at center $\mathbf{B}_{\min }=0.42 \mathrm{~T}$, at extraction $\mathbf{B}_{\text {ext }}=0.5 \mathrm{~T}$.

\subsection{Charge breeding of ${ }^{23} \mathrm{Na}$}

Hereafter, the experiments were performed with helium as buffer gas in ECR charge breeder, which is observed to be the best for optimizing the charge breeding efficiencies of high charge states of $\mathrm{K}$ and $\mathrm{Na}$ [7]. Please refer to table 2 for typical values used for charge breeding of Na. The thermionic ion gun was used to produce the ${ }^{23} \mathrm{Na}^{+}$beam at an extraction voltage $\mathrm{V}_{1+}=+(20$ $\mathrm{kV}+\Delta \mathrm{V})$. The $1+$ ions are mass selected by the $90^{\circ}$ dipole and focused through the set of slits where the emittance $(4 \sigma)$ is defined as $30 \pi . \mathrm{mm}$.mrad (non-normalized). The beam is focused again by the electrostatic triplet and injected into SPIRAL1 charge breeder with an intensity of $550 \mathrm{nA}$. The high voltage applied to the charge breeder $\left(\mathrm{V}_{\mathrm{CB}}\right)$ is $20 \mathrm{kV}$.

Table 2. Parameters used for Charge breeding of ${ }^{23} \mathrm{Na}$.

\begin{tabular}{|l|c|}
\hline \multicolumn{1}{|c|}{ Parameters } & Charge breeding of ${ }^{\mathbf{2 3}} \mathbf{N a}$ \\
\hline Magnetic field at injection $\left(\mathrm{B}_{\text {inj }}\right)$ & $1.41 \mathrm{~T}$ \\
\hline Magnetic field at injection $\left(\mathrm{B}_{\min }\right)$ & $0.42 \mathrm{~T}$ \\
\hline Magnetic field at injection $\left(\mathrm{B}_{\mathrm{ext}}\right)$ & $0.91 \mathrm{~T}$ \\
\hline RF power (Watts) & $540 \mathrm{~W}$ \\
\hline
\end{tabular}

The $\mathrm{V}_{1+}$ is regulated through $\Delta \mathrm{V}$ with respect to the $\mathrm{V}_{\mathrm{CB}}$ in order to optimize $1+$ injection. The charge bred ions are extracted through extraction system and the required charge state is selected by the bending dipole magnet. Finally, the charge breeding efficiency is calculated as

$$
\epsilon_{c b}=\frac{1}{I_{1+}} \sum_{q=1}^{q_{\max }} \frac{\Delta I_{q+}}{q^{+}}
$$

Where, $\Delta I_{q_{+}}$is difference in extracted beam currents with and without $1+$ injection, $q^{+}$is the required ion charge state and $I_{1_{+}}$is the injected beam intensity. In this way, the charge breeding efficiencies calculated for ${ }^{23} \mathrm{Na}^{2+},{ }^{23} \mathrm{Na}^{7+},{ }^{23} \mathrm{Na}^{8+}$ and ${ }^{23} \mathrm{Na}^{9+}$ are $0.82 \%, 3.23 \%, 3.57 \%$ and $1.2 \%$, respectively. The $\Delta \mathrm{V}$ was tuned between $6 \mathrm{~V}$ and $8 \mathrm{~V}$. The $\Delta \mathrm{V}$ value for maximum achieved efficiency is same for high charge states of sodium, which was similar when compared with results obtained for sodium with LPSC PHOENIX charge breeder [8]. 


\subsection{Effect of deceleration tube position on charge breeding efficiency}

In SPIRAL1 charge breeder, the charge breeding efficiencies for ${ }^{23} \mathrm{Na}^{8+}$ were recorded at different positions of the deceleration tube. Observing the geometry sub-plot in figure 4, the optimum position is found at a distance of $21 \mathrm{~mm}$ from the injection plug of $\mathrm{CB}$. The ${ }^{23} \mathrm{Na}^{+}$beam measured downstream from the charge breeder was stable at the optimum position and became unstable when the tube was moved to the ends. This effect was clearly marginal during the charge breeding test performed with SP1 charge breeder at the 1+/n+ LPSC test bench [4]. This underlines the sensitivity of injection ion optics in LEBT which can affect the injection efficiency and consequently, the charge breeding efficiency.

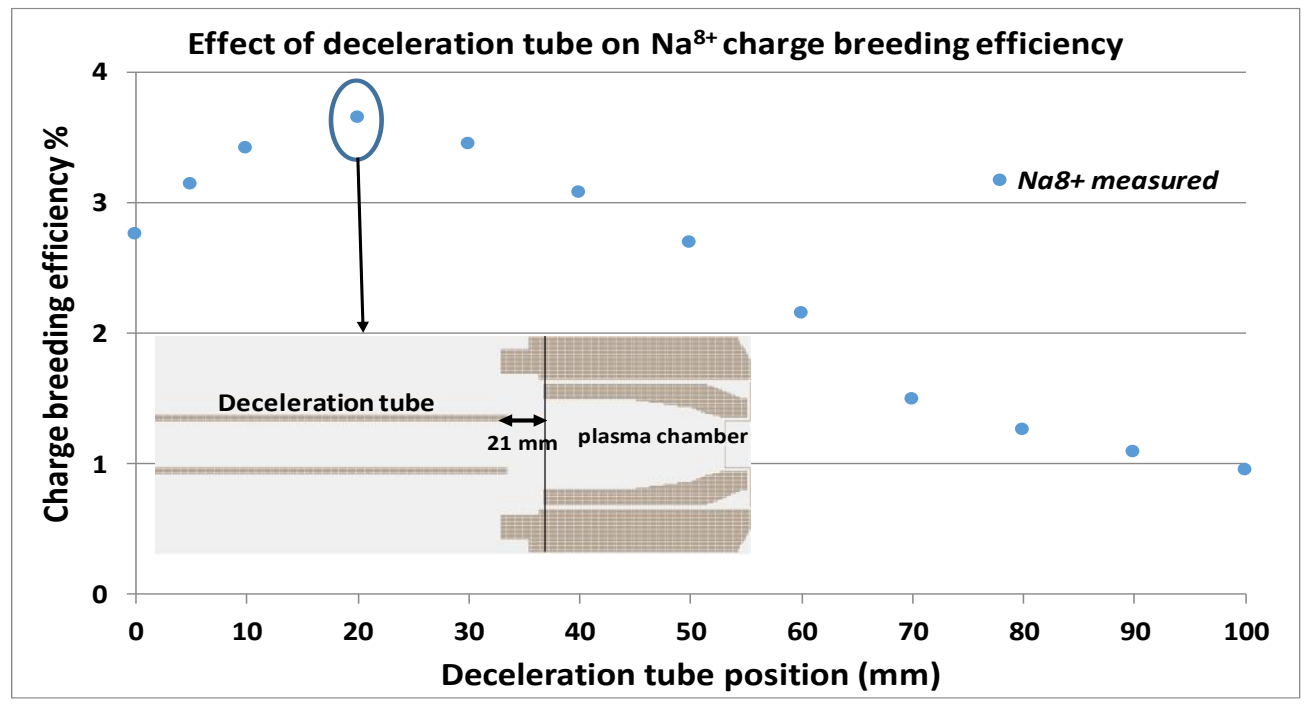

Figure 4. Effect of deceleration tube position on $\mathrm{Na}^{8+}$ charge breeding efficiency. At $0 \mathrm{~mm}$, the position of the tube is inside the charge breeder. Measured $\mathrm{Na}^{8+}$ curves are normalized to $13.65 \%$.

\subsection{Effect of injected beam emittance on charge breeding efficiency}

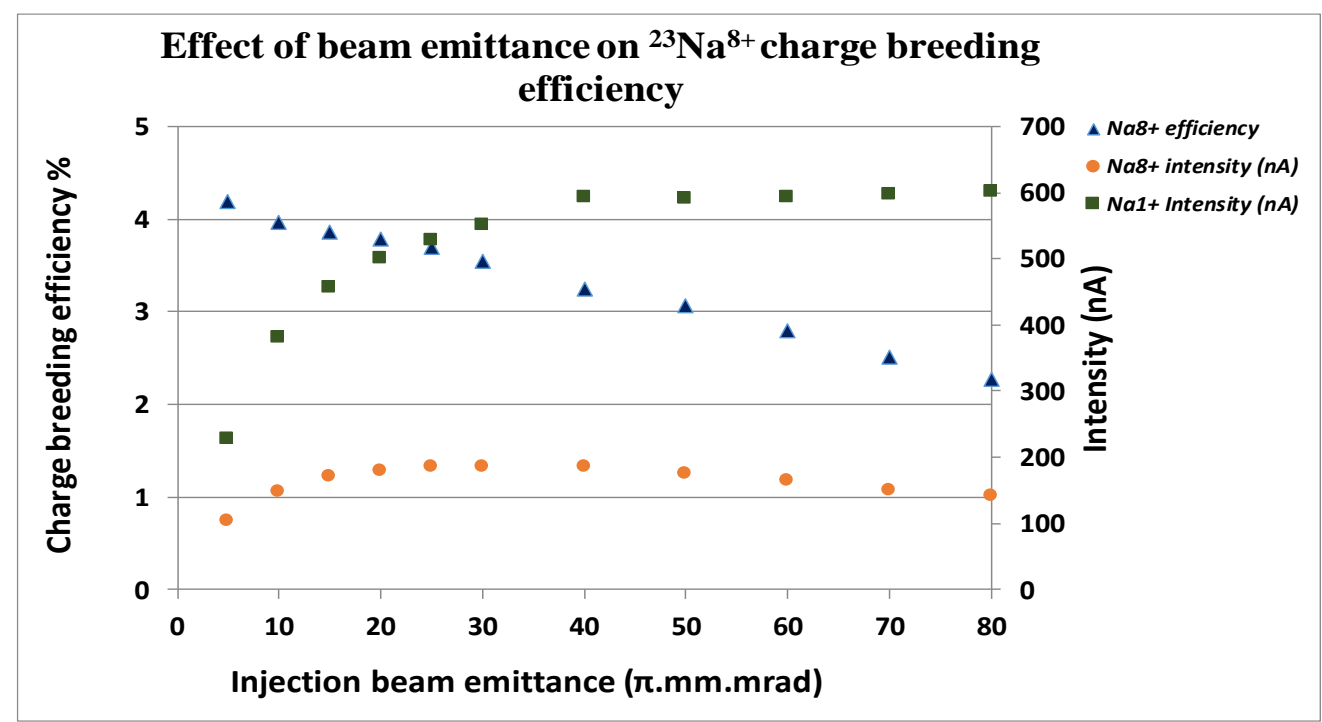

Figure 5. Effect of beam emittance on $\mathrm{Na}^{8+}$ charge breeding efficiency. 
The $20 \mathrm{kV}{ }^{23} \mathrm{Na}^{+}$beam was injected into SPIRAL1 charge breeder with an intensity of 550nA. $\Delta \mathrm{V}$ was optimized in order to reach a Charge breeding efficiency of $3.53 \%$ on ${ }^{23} \mathrm{Na}^{8+}$. The injection beam emittance defined for this measurement is $30 \pi \mathrm{mm} \cdot \mathrm{mrad}$ (non-normalized). Later, the injected beam emittance was then varied from $5 \pi \mathrm{mm}$.mrad to $80 \pi \mathrm{mm}$.mrad by adjusting a set of slits between the $1+$ source and the $\mathrm{CB}$. The intensities of $\mathrm{Na}^{1+}$ and $\mathrm{Na}^{8+}$ beam is recorded at the Faraday cup $\left(\mathrm{FC}_{1+}\right)$ placed before the injection optics of the charge breeder and Faraday cup $\left(\mathrm{FC}_{\mathrm{n}+}\right)$ placed in $\mathrm{n}+$ line after the mass analyzer. Figure 5 represents the ${ }^{23} \mathrm{Na}^{8+}$ charge breeding efficieny and $1+/ 8+$ intensities as a function of injected beam emittance. At low emittance $(<40$ $\pi . \mathrm{mm} . \mathrm{mrad})$, the $1+$ beam that is actually injected into the charge breeder is collimated by the set of slits, which represents a significant drop in 1+ beam current. Consequently, the $\mathrm{n}+$ current has shown a significant drop at low emittance which is due to the injection of low 1+ intensity into the charge breeder. At higher emittance ( $>50 \pi . \mathrm{mm}$.mrad), a small drop in $\mathrm{Na}^{8+}$ beam currents has been observed. It suggests that perhaps increased ion losses in the injection region affecting the injection efficiency. The observed charge breeding efficiency trends steadily decrease with increase in emittance.

\section{Numerical simulation studies on ECR charge breeder}

\subsection{Simulation description}

Numerical simulations were performed to evaluate the ion losses during the transport of $1+$ beam through the charge breeder with and without a simplified model of the ECR plasma. These cases correspond with the situations where the $\mathrm{CB}$ is operated in shooting-through mode and charge breeding mode. Figure 6 represents the geometry of Low Energy Beam Line from the beam slits position to the extraction system of the charge breeder was implemented in a particle tracking code called SIMION 3D [9]. The geometry includes the injection optics (electrostatic quadrupole lens, deceleration tube), SPIRAL1 charge breeder and extraction system. The 3D magnetic field of the $\mathrm{CB}$ is externally calculated from RADIA and given as an input for the simulations. The electric fields are calculated in SIMION by applying potentials to the geometry electrodes. For initiating the ions in the simulation, a Matlab code has been developed, which can generate the initial ions using beam properties (twiss parameters and transversal co-ordinates) obtained from TraceWin code $(\alpha=-2.3019, \beta=0.7715)$. The particles created in the Matlab code should satisfy the following phase space relations in order to be a part of the beam.

$$
\begin{aligned}
& \gamma x^{2}+2 \alpha x x^{\prime}+\beta x^{\prime 2} \leq \epsilon \\
& \gamma y^{2}+2 \alpha y y^{\prime}+\beta y^{\prime 2} \leq \epsilon
\end{aligned}
$$

Also, the energy and mass of $1+$ ions can be changed in the code to simulate the ions with different mass and energies. The input file is written as ion mass, ion charge state, position of particle in $\mathrm{x}, \mathrm{y}, \mathrm{z}$, angles $\left(x^{\prime}, y^{\prime}\right)$ which satisfy the above relations and ion energy. In this way, the starting conditions for the simulation are defined.

\subsection{Ion transport through the charge breeder without plasma}

The transmission efficiencies obtained for ${ }^{39} \mathrm{~K}^{+}$and ${ }^{23} \mathrm{Na}^{+}$ions are discussed in the previous section. When the high voltage of CB is OFF (shooting-through), transmission efficiencies were recorded. 


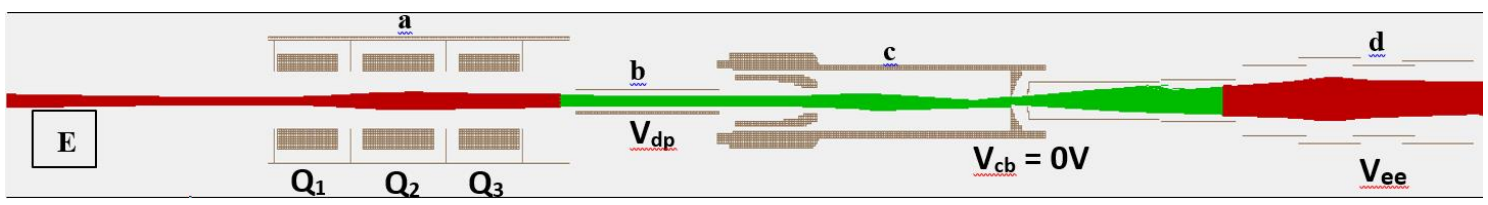

Figure 6. The geometry of electrostatic quadrupole lens (a), decleration tube (b), ECR charge breeder (c) and extraction einzel lens (d) used in the ion transport simulation. At the point $\mathrm{E}, 10000{ }^{39} \mathrm{~K}^{+}$ions are initiated with the energy of $10 \mathrm{keV}$ and simulated through ECR charge breeder. The total ions extracted from charge breeder are counted after the extraction system.

Later, the potential of $\mathrm{CB}$ is ramped from $1-15 \mathrm{kV}$ and observed an increase (also decrease) in efficiency. To understand these fluctuations, ${ }^{39} \mathrm{~K}^{+}$and ${ }^{23} \mathrm{Na}^{+}$ions at energy of $10 \mathrm{keV}$ and $15 \mathrm{keV}$ are simulated through the $\mathrm{CB}$ with an emittance of $50 \pi \mathrm{mm} . \mathrm{mrad}$ (non-normalized) at the characterization point $\mathrm{E}$. The emittance used for this case is similar to the experimental ones. Figure 6 shows the simulation of ${ }^{39} \mathrm{~K}^{+}$ions transported through the $\mathrm{CB}$. The ion losses are monitored in the simulation by writing a dedicated Lua user program. In the simulation, z-axis is taken as reference axis. The program records the particle count before and after the injection optics, charge breeder and extraction system. The transmission efficiency is calculated by considering the ratio between the total extracted particles from the charge breeder and total particles injected into the charge breeder. In both cases $\left(\mathrm{K}^{+}\right.$and $\left.\mathrm{Na}^{+}\right)$, the simulation has shown that up to $20 \%$ of $1+$ ions were lost at the extraction aperture.

\subsection{Ion transport through the Charge Breeder plasma}

In this case, the numerical simulation basically simulates the ion transport through a simplified plasma model introduced in the charge breeder, which allows to reproduce the trends of experimental $\Delta \mathrm{V}$ curves. The $\mathrm{CB}$ geometry was modified by including a thin cylindrical symmetry electrode of plasma sheath $\left(\mathrm{V}_{\mathrm{p}}\right)$, ECR zone $\left(\mathrm{V}_{\mathrm{ECR}}\right)$ and potential dip $\left(\mathrm{V}_{\mathrm{dip}}\right)$ inside the ECR zone. Fixed potentials are given to these electrodes to approximate the potential distribution in plasma volume. The sheath created by the plasma is considered to be contained within the two maxima of the magnetic field, thus the contour is extended from the maximum at the injection up to the extraction electrode. The distance between the wall and sheath is $1 \mathrm{~mm} /$ grid cell. The geometry of ECR zone is located at minimum-B structure formed by the three solenoids. It has been argued [10] that the potential dip should be formed inside the ECR zone and simulations were performed regarding the processes in ECRIS [11], where the potential dip is confined inside the ECR zone. From these references, the potential dip is implemented inside the ECR zone in order to see its effect on trends of $\Delta \mathrm{V}$ curves. The potentials applied to the sheath $\left(\mathrm{V}_{\mathrm{p}}\right)$ and $\mathrm{ECR}$ zone $\left(\mathrm{V}_{\mathrm{ECR}}\right)$ are always equal in all cases in order to confine the potential dip inside the resonance zone.

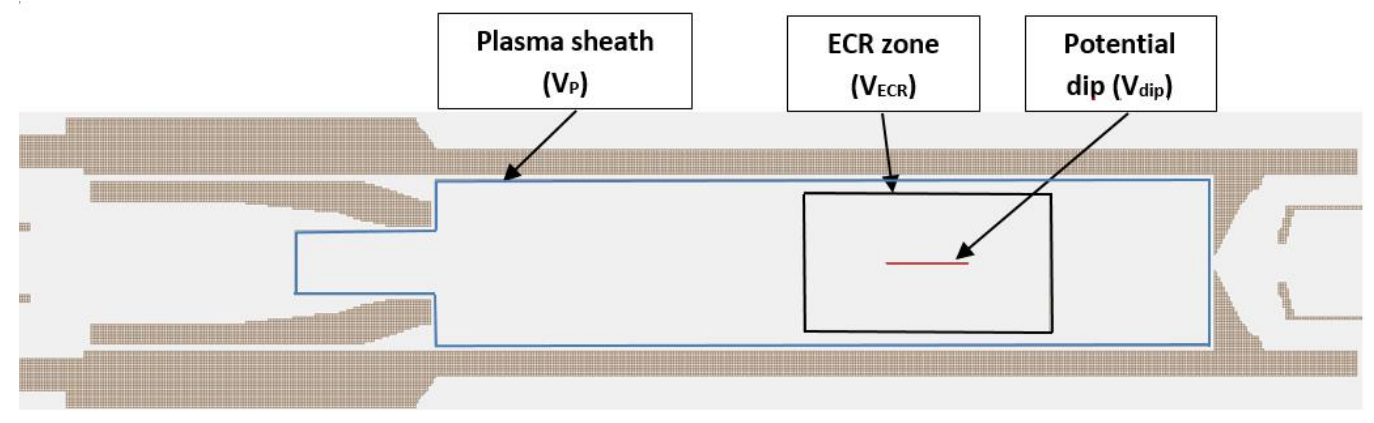

Figure 7. The geometry of ECR charge breeder with the ECR plasma features. 
To consider the deceleration of $1+$ ions, the potentials applied to the geometry of plasma potential $\left(\mathrm{V}_{\mathrm{p}}\right)$ and ECR zone $\left(\mathrm{V}_{\mathrm{ECR}}\right)$ is higher than the potential of charge breeder $\left(\mathrm{V}_{\mathrm{cb}}\right)$. The potential dip is always considered as $\mathrm{V}_{\text {dip }}=\mathrm{V}_{\mathrm{ECR}}-1 \mathrm{~V}$. The plasma potential of $10 \mathrm{~V}$ has been chosen in order to reproduce the $\Delta \mathrm{V}$ curves. However, based on experimental optimum $\Delta \mathrm{V}$ results we know that the plasma potential in reality is lower, most likely below $8 \mathrm{~V}$. But in order to simulate the trends, this discrepancy should not be critical. Ions are initiated with an emittance of $30 \pi . \mathrm{mm}$.mrad at energy of $20 \mathrm{keV}$. The $\Delta \mathrm{V}$ curves for $\mathrm{Na}^{1+}$ in the simulation are defined as the potential difference between the charge breeder and injection energy of 1+ions. Besides, the idea of simulating $\mathrm{Na}^{2+}$ ions through the simplified plasma model has been implemented to see the evolution of $\mathrm{Na}^{2+} \Delta \mathrm{V}$ curves. As the simplified plasma model presented in this paper does not include collisions or other interactions between the ions, the $\mathrm{Na}^{2+}$ ions are initiated at three different locations inside the plasma chamber. A dedicated Lua program has been written to initiate the $\mathrm{Na}^{2+}$ after overcoming the potential barrier, at the boundary of ECR surface and inside the ECR surface. Using this program, the mass and charge of an ion can be changed without disturbing the kinetic energy of ions. The efficiency is finally calculated at the end of extraction system as the ratio between number of particles extracted and number of particles injected.

\section{Simulation Results and discussion}

With the transmission simulations (CB off), Transmission efficiencies of $84 \%$ and $79 \%$ for $\mathrm{K}^{+}$ and $\mathrm{Na}^{+}$were recorded after the extraction system, which are very similar to the experimental results reported in the previous sections. From the observations, $100 \%$ transmission has been achieved up to the entrance of CB. The ion losses were observed only at the extraction aperture. Ion losses significantly increase at extraction aperture with increase in $\mathrm{V}_{\mathrm{cb}}$.

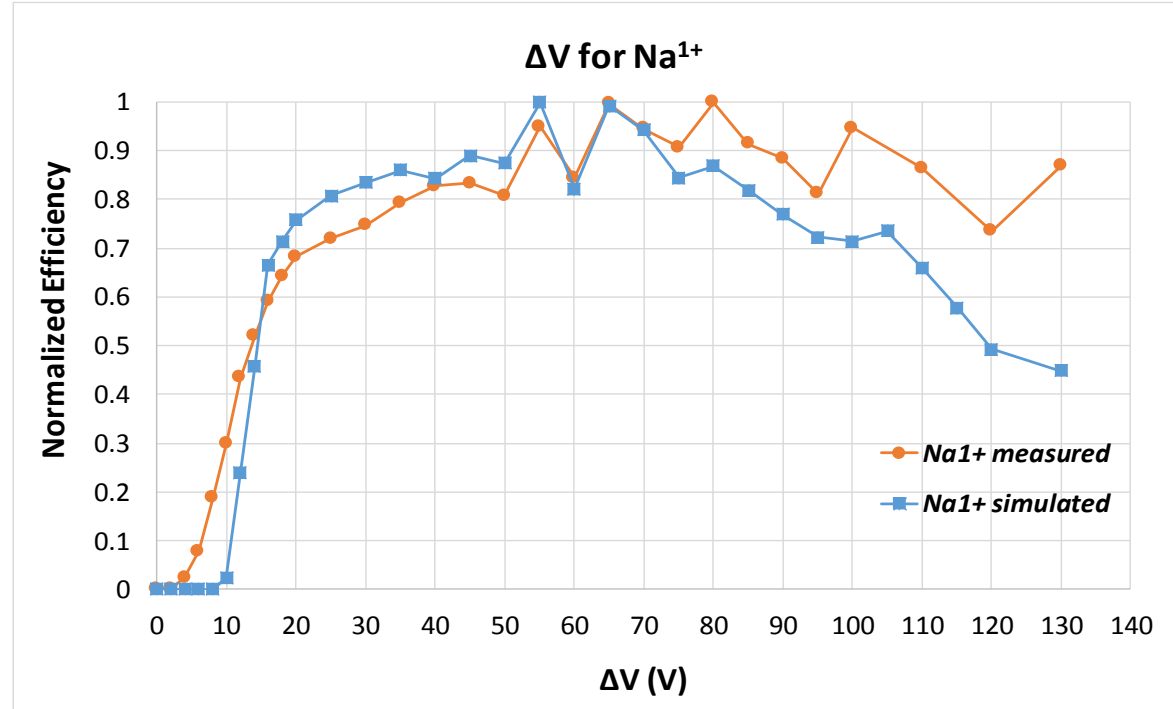

Figure 8. Normalized efficiency of $\mathrm{Na}^{+}$as a function of $\Delta \mathrm{V}$. Measured and simulated curves are normalized to 1 from $28 \%$ and $24 \%$.

The second part of the numerical simulation concerned the extraction of $\mathrm{Na}^{+}$and $\mathrm{Na}^{2+}$ through CB plasma volume. From the observations, the recorded simulated curve seems to shift towards higher $\Delta \mathrm{V}$ when compared with experimental curves. In order to find the best agreement 
with the experimental curves, the emittance of $1+$ beam has been varied to $20 \pi$.mm.mrad by keeping the same twiss parameters. At low $\Delta \mathrm{V}$, most of the ions are reflected back to the deceleration tube due to positive plasma potential (e.g. $85 \%$ of ions with $\Delta \mathrm{V}$ between $9-10 \mathrm{~V}$ ). When $\Delta \mathrm{V}$ is at $55 \mathrm{~V}$, the maximum of the $\mathrm{Na}^{+}$is obtained, and the ion losses at the entrance of plasma chamber significantly decreased to $15 \%$. In case of $\mathrm{Na}^{2+}$, the $\Delta \mathrm{V}$ curves are recorded at three different locations as explained in the previous section. The $\Delta \mathrm{V}$ curves of $\mathrm{Na}^{2+}$ ions which are initiated at the boundary of ECR surface agree with the experimental data and presented in Figure 9. The simulation seems to significantly over-estimate the efficiency compared to the measured values. This is probably due to the very simplified $1+$ to $2+$ conversion model implemented in the simulations.

During the simulations, periodic oscillations were observed after 50V and higher, which was also observed in $\mathrm{Na}^{+}$experimental curves. These discrepancies observed in the simulations revealed that the $1+$ (and maybe $2+$ ) beam traversing through the plasma chamber exhibits a periodic focusing/defocusing behavior due to the solenoid field [12], and consequently the phase of this motion at the extraction aperture influences the transmission. As this motion is ion energy dependent, varying $\Delta \mathrm{V}$ or $\mathrm{V}_{\mathrm{cb}}$ influences the phase at the extraction aperture and therefore the transmission. Thus this phenomenon can explain some of the features seen in the simulation results (oscillating transmission). As similar oscillations are present in measured data, it suggests that perhaps similar effects takes place in reality. Also, the potential dip is varied by few volts and its effect was found to be negligible for the trends of $\Delta \mathrm{V}$ curves.

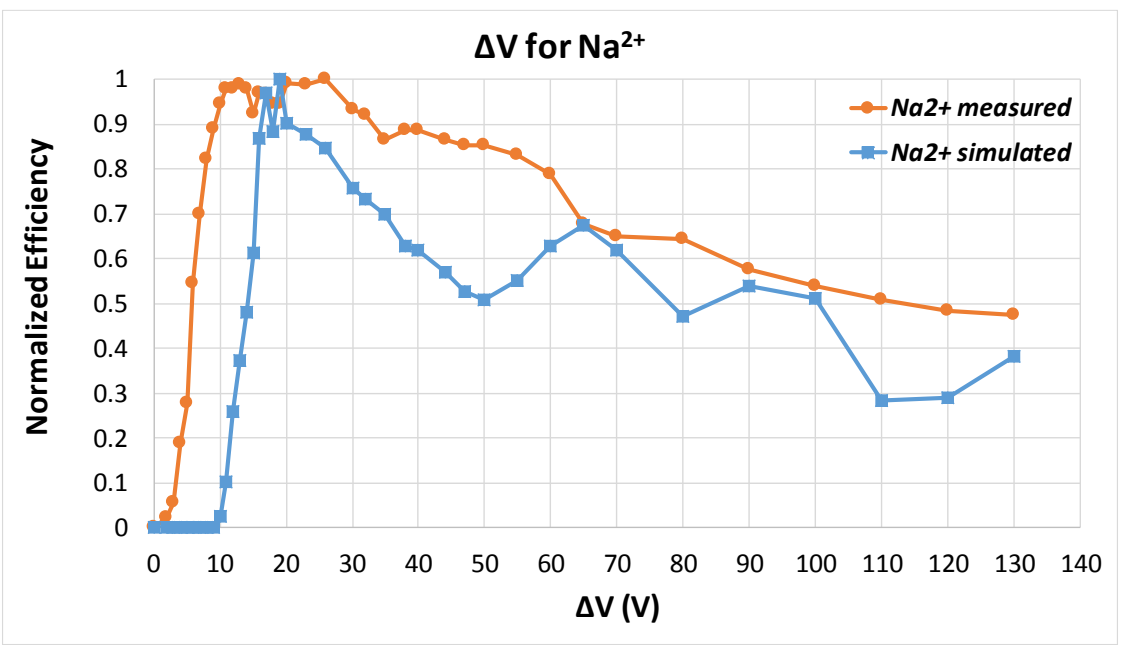

Figure 9. Normalized efficiency of $\mathrm{Na}^{2+}$ as a function of $\Delta \mathrm{V}$. Measured and simulated curves are normalized to 1 from $0.9 \%$ and $21 \%$.

\section{Summary and perspectives}

Simulations revealed that the ion losses at the extraction aperture are mostly due to the beam oscillations in the charge breeder. The transmission efficiencies obtained from the simulations show good agreement with the experimental results. The $\Delta \mathrm{V}$ plots for $\mathrm{Na}^{+}$and $\mathrm{Na}^{2+}$ obtained from a rough analysis performed with the simplified ECR plasma model has shown similar trends when compared with the experimental curves. In the future, the simulation model presented in this paper will be improved by distributing the $\mathrm{Na}^{2+}$ ions randomly in the core of ECR plasma instead of using a conversion from $1+$ to $2+$ at a fixed axial position. Simultaneously, the $\Delta V$ plots for $\mathrm{Na}^{+}$ 
and $\mathrm{Na}^{2+}$ will be calculated with a numerical simulation code called MCBC (Monte Carlo Charge Breeding Code) which can model the capture and charge breeding of a beam of ions injected into plasma [13]. The $\Delta \mathrm{V}$ plots obtained from SIMION 3D and MCBC will be compared for further investigation regarding the $1+$ capture process by ECR plasma. To complete this analysis, additional experimental data will be gathered from $\mathrm{Mg}$ (using He buffer gas) and $\mathrm{K}$ (using $\mathrm{He}$ and $\mathrm{O}_{2}$ buffer gas) charge breeding efficiency (also charge breeding time) measurements. This approach can show a possible way to determine the tendency of charge breeding parameters which influences the 1+ ion capture efficiency by the ECR plasma.

\section{References}

[1] O. Kamalou et al., GANIL Operation Status and New Range of Post-Accelerated Exotic Beams, in Proceedings of the $13^{\text {th }}$ International Conference on Heavy Ion Accelerator Technology, pp.20-22 (2015).

[2] M. Duval et al., New compact cyclotron design for SPIRAL, in Proceedings of the $14^{\text {th }}$ International Workshop on Magnet Technology, MT-14 Tampere, Finland, June 11-16 (1995).

[3] P. Delahaye et al., Charge breeding of light metallic ions: Prospects for SPIRAL, Nuclear Instruments and Methods in Physics Research, A 693104 (2012).

[4] L. Maunoury et al., SPIRAL1 Charge Breeder: Performances and Status, in Proceedings of the $22^{\text {nd }}$ International Workshop on ECR Ion Sources, Busan, South Korea, $28^{\text {th }}$ August- $1^{\text {st }}$ September (2016).

[5] RADIA magnetic field simulation code, http://www.esrf.eu/Accelerators/Groups/InsertionDevices/Software/Radia

[6] http://irfu.cea.fr/dacm/logiciels/

[7] L. Maunoury et al., Charge breeder for the SPIRAL1 upgrade: Preliminary results, Review of Scientific Instruments, 87, 02B508 (2015).

[8] T. Lamy et al., in Proc. of the $18^{\text {th }}$ International Workshop on ECR Ion Sources, Chicago, Illinois, US, September 15-18, 2008.

[9] SIMION Ion and Electron Optics Simulator, http://www.simion.com (ScientificInstrumentServices,Inc.(SIS))

[10] V. Mironov et al., Numerical model of electron cyclotron resonance ion source, Physical review special topics-accelerators and beams 18, 123401 (2015).

[11] B. Cluggish et al., Simulation of parameter scaling in electron cyclotron resonance ion source plasmas using the GEM code, Review of Scientific Instruments, 81, 02A301 (2010).

[12] V. Kumar, Understanding the focusing of charged particle beams in a solenoid magnetic field, American Journal of Physics, 77. 737 (2009).

[13] JS. Kim et al., Ion beam capture and charge breeding in electron cyclotron resonance ion source plasmas, Review of Scientific Instruments, 78, 103503 (2007). 